\title{
U-shaped masking functions in visual back ward masking: Effects of target configuration and retinal position ${ }^{1}$
}

\author{
ALAN L. STEWART, 2 YORK UNIVERSITY \\ AND DEAN G. PURCELL, UNIVERSITY OF TORONTO
}

\begin{abstract}
Printed letters were found to become increasingly susceptible to visual backward masking when presented to one side of the point of fixation. All masking was found to be a $u$-shaped function of the time interval separating the offset of the target from the onset of the masking figure. The interval at which maximum masking was observed, as well as the amount of masking observed, varied with the target-mask configuration studied. More masking was found when the ISI field was lighted than when it was not.
\end{abstract}

A complex stimulus is sometimes conceived of as being made up of an array of simpler elements. When the stimulus consists of a row of printed letters, each letter is considered an element of the more complex array. Recent studies have attempted to determine whether the elements comprising such a complex visual stimulus are processed in series or in parallel. Processing of each element of the stimulus is hypothesized to take place item by item in serial processing so that as the array increases in size, processing time should increase. In parallel processing, each element is hypothesized to be processed simultaneously. The time for parallel processing of the stimulus should be constant, within the S's ability to handle a stimulus of a given complexity. For both formulations, it has seemed reasonable to decide between serial and parallel processing of a briefly presented stimulus, in park, on the basis of whether or not there is an increase in response time with an increase in the number of elements in the visual array (Sternberg, 1967).

A second approach has been to employ a backward-masking paradigm (Neisser, 1967; Raab, 1963; Werner, 1935). A visual target presented for periods as short as $1 \mathrm{msec}$ can be identified correctly. If this target is followed by a second visual stimulus, then, under some circumstances, the target can no longer be identified. This intereference with the perception of a target by a subsequent stimulus is referred to as "backward masking." It has been assumed that the mask disrupts the processing of the first stimulus. The minimum duration of the processing sequence has been inferred to be the time between the onset of the target stimulus and the greatest delay at which the masking stimulus still has an effect (Weisstein, 1966). The decision between parallel and serial processing is similar to that used in the studies measuring response times. As the number of letters in the complex array increases, the masking stimulus should be effective at longer delays if each letter is processed serially. On the other hand, the longest delay at which the mask is effective should remain constant if the elements are processed simultaneously.

When the per cent of times the target is correctly identified is plotted against the delay interval of the mask, two types of curves have been reported (Kolers, 1962). Under some conditions, the effectiveness of the mask decreases as a function of increasing delay; under different conditions, the mask first becomes more effective as the delay increases and then becomes less effective at still greater delays. The point in time at which the greatest masking takes place is of interest in its own right. Mathematical models of masking have addressed themselves to predicting the shape and properties of temporal masking curves (Weisstein, 1968; Landahl, 1967). Still other theoretical speculations that suggest that perceptual processing is confined within blocks of discrete quantial units have relied, in their analysis, on data from masking studies (Leibovic, 1965, 1968).

Evidence has been presented that supports parallel processing (Neisser, 1963) as well as serial processing of each element (Sperling, 1963; Averbach \& Coriell, 1961). Other studies have reported that processing is not done in a strictly parallel or serial fashion (Weisstein, 1966). All of these studies have one implicit assumption in common: It is assumed that as more elements are added to the visual array, each element continues to have an equal impact on the visual system. In each of the above experiments, however, the data were gathered in such a way that the position occupied by each element was ignored in the data analysis.

Two additional criticisms have been made of the masking paradigms used by Weisstein (1966). First, it has been suggested that the $u$-shaped functions reported by Weisstein may be an artifact of using an annular masking figure both as a masking stimulus and as an indicator of which element of a multiple-item visual array the $S$ is to report (Eriksen, Collins, \& Greenspon, 1967). Such a criticism, however, is restricted to masking functions obtained with visual arrays where two or more letters are presented simultaneously. It does not apply to those u-shaped functions obtained by Weisstein when only one target letter was presented at random to one of the number of different possible retinal loci. For such single-item presentations, a second, more general criticism has been made: In Weisstein's experiments, each single item was presented randomly, across ISI, to either 1 of 4 or 1 df 8 different retinal loci without regard for differences in retinal sensitivity. On this basis, it has been argued that u-shaped functions obtained with only one target item are the results of an inadvertent allocation of the target to less sensitive retinal loci at intermediate ISI values (Eriksen, Collins, \& Greenspon, 1967).

Because of the theoretical interest of $\mathrm{u}$-shaped masking functions, it is important to determine if they are the result of the temporal interaction of the mask with the target or if they are artifacts of the specific experiments in which they have been found. If the u-shaped functions are artifacts of failing to counterbalance retinal locus of the target presentation across ISI values, they should not be obtained when retinal locus of item presentation is held constant. The following experiments were designed to study the identification of target letters under conditions of backward masking, with retinal locus of presentation held constant for all ISI values.

\section{EXPERIMENT 1}

One of two black, printed letters was briefly presented via a tachistoscope (Scientific Prototype GB) and followed by a masking stimulus. In typical masking experiments, the $S$ fixates on a point located at the center of the stimulus field, and the stimuli are presented either at the point of fixation or at some distance from that point. In this experiment, a slightly different procedure was followed. The letter to be masked was always presented in the center of the illuminated stimulus field (182-min square), letter contrast ratio 0.12 . Depending on the experimental 


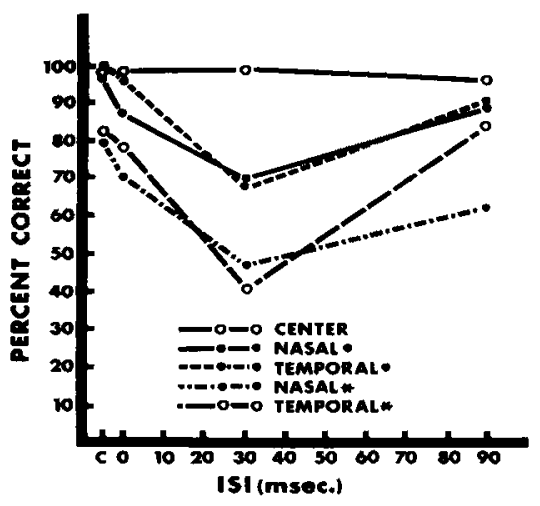

Fig. 1. The per cent correct identification for the target pair " $D$ " and " $O$," corrected for chance. The fixation point was located at one of five positions: the point at which the target was presented, and at points $36.6 \mathrm{~min}(\cdot)$ or $103.8 \mathrm{~min}(*)$ nasal and temporal of the target-presentation point.

condition, the $S$ fixated on the point at which the letter appeared or on a point to one side of the center of the stimulus field. The per cent of times the $S$ correctly identified the masked letter served as an index of the letter's susceptability to masking and indicated how easily recognized the letter would be when presented to a corresponding retinal locus as part of a larger visual array.

Five points of fixation were studied: central fixation, and points 38.4 and $105.6 \mathrm{~min}$ of arc nasal and temporal of the center point of the stimulus field. Time and luminance parameters were chosen that were comparable to those used by Weisstein and Haber (1965). Target duration was $20 \mathrm{msec}$, and mask duration was $50 \mathrm{msec}$. Four delay intervals were studied: concurrent, where the target and the mask onset were simultaneous, but where the mask outlasted the target by $30 \mathrm{msec}$; an interstimulus interval (ISI) of $0 \mathrm{msec}$, where the target offset was immeidately followed by the mask onset; and ISIs of 30 and $90 \mathrm{msec}$, measured from target offset to mask onset. The adaptation field, target-presentation field, lighted ISI, and masking fields were illuminated at $10 \mathrm{ft}-\mathrm{L}$.

Two different masking conditions were paired with each of the above experimental conditions. Under one condition, no masking figure was present in the masking field, although the target offset was followed by a homogeneously illuminated stimulus field, the duration of which was equal to the appropriate ISI-interval-plus-mask duration. Under the second condition, a small ring-shaped figure was introduced into the masking field. During concurrent presentation, the ring appeared to encircle the target snugly (ring diam, $36.6 \mathrm{~min}$; strip width, $2.4 \mathrm{~min}$ ). The letters " $D$ " and "O" were used as targets (letter heights, 28.8 and 28.8, and letter widths, $24 \mathrm{~min}$ and $28.8 \mathrm{~min}$, respectively; strip width, $4.8 \mathrm{~min}$ ).

Five well-trained psychophysical observers served as Ss. All Ss had participated in a large number of backward-masking experiments. Ss viewed the adaptation field for $10 \mathrm{~min}$ preceding the trials and were given four practice trials under each experimental condition just before it was run. After carefully fixating on the appropriate point, Ss initiated the target presentation by depressing a thumb switch. All responses were made as forced choices. Viewing was monocular, right eye. Data were gathered in blocks of 25 trials with two replications, for a total of 50 responses per $S$ under each condition. Conditions were randomized within replications.

\section{Results and Discussion}

Masking occurred only when the mask contained the annular masking figure. With the masking figure present, the percentage of errors made by the $S$ increased as the fixation point was located at successively greater distances from the point of target presentation $[F(4,16)=21.65, p<.01]$. Except under central fixation, masking at each fixation point was a u-shaped function of the delay between target offset and masking-figure onset $[F(3,12)=14.54$, $\mathrm{p}<.01]$. Virtually no masking took place under central fixation, as Fig. 1 illustrates [interaction of Fixation by ISI yields $F(12,48)=2.90, p<.01]$. The amount of masking appeared similar for comparable nasal and temporal fixation points.

The assumption that similar targets have an equivalent impact on the visual system, independent of their position within a complex array, was not supported by the data. The data did not indicate, however, whether or not the configuration of the target itself influenced its susceptibility to backward masking. The letters used as targets in masking experiments are often chosen because their outlines are similar, causing the letters to be easily confused with each other. It is not clear if pairs of letters that are easily confused with each other are also equally sensitive to masking effect. Nor is it clear if these pairs of letters also interact with the masking figure in the same way. To examine the effects of a target's contour on its susceptibility to masking, pairs of letters whose configuration differ from each other were used as targets under identical masking conditions.

\section{EXPERIMENT 2}

Three different pairs of target letters were studied. Both the target pair " $D$ " and "O" and the target pair " $A$ " and " $T$ " were selected because they had been extensively used in ba ckward-masking experiments-the pair " $D$ " and " $O$ " by Weisstein and Haber (1965) and Weisstein (1966), and the pair " $A$ " and " $T$ " by Eriksen and Collins (1964), Eriksen (1966), and Eriksen, Collins, and Greenspon (1967). The third target pair consisted of the letter "C," oriented so that the break in its defining contour was directed at the top or the bottom of the presentation field (letter height, $24 \mathrm{~min}$ of arc, and width, 28.8 min; strip width, $4.8 \mathrm{~min}$ ). Two factors determined its selection. The configuration of the " $\mathrm{C}$ " is similar to that of the letter " $U$," which, along with the letters " $A$ " and " $T$," is the third target letter used by Eriksen and his colleagues. Unlike the letter " $U$," the letter " $\mathrm{C}$ " has its contours equidistant from the adjacent contour of the masking figure and so more closely meets the criterion of close spatial proximity of the target and the mask required by Weisstein's model of metacontrast (1968).

The experimental conditions were identical with those in Experiment 1, except for the following: A single fixation point was located $105.6 \mathrm{~min}$ of arc, nasal to the point at which the targets were presented; the annular masking figure was always included in the masking field; its onset was studied for concurrent presentations and for time delays of 0,5 , $10,20,30$, and $70 \mathrm{msec}$ following the offset of the target. One hundred observations by each of the five Ss were

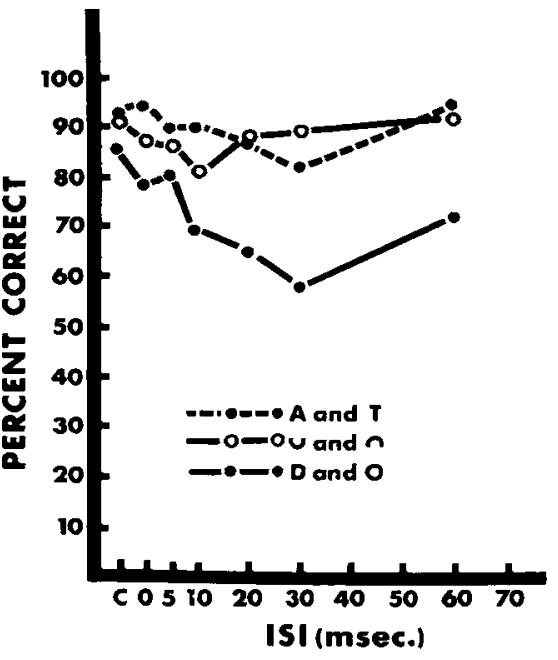

Fig. 2. Per cent correct identification for the target pairs " $D$ " and " $O$," " $A$ " and "T," and " $C$," corrected for chance. The fixation point was $103.8 \mathrm{~min}$ of arc nasal of the point at which the target was presented. 
gathered under each experimental condition.

\section{Results and Discussion}

Masking for each of the target pairs was a u-shaped function of the ISI, as Fig. 2 illustrates $[F(6,24)=6.60, p<.001\}$. The amount of masking varied with the pair of letters used as targets $[F(2,8)=24.86$, $p<.001]$. Taken in conjunction with the results of Experiment 1, the data suggest that the amount of masking observed when using a backward-masking paradigm is influenced by both the configuration of the targets and the retinal position at which the targets are presented. It is possible that each individual target letter, depending on its position within a complex array, will yield different masking functions under the same masking conditions. With a complex array, made up of a number of different letters, the average amount of masking cannot be compared arbitrarily with the amount of masking achieved with a second array, made up of a different set of letters.

A second feature of the data indicates a more serious obstacle to using data obtained through masking experiments in studying information processing. The time delay at which maximum masking occurs might be assumed to be determined by the rate at which visual information is processed. But the results of Experiment 2 indicate that the point at which maximum masking is observed is influenced by the configuration of the target. The point of maximum masking-the u-minimum-for the letter " $C$ " is about $10 \mathrm{msec}$, while the u-minimum for the other letter pairs occurs somewhere between 30 and $70 \mathrm{msec}$ [ISI by Target yields $F(12,48)=2.60$, $\mathrm{p}<.025]$. The experimental conditions are identical for all the letter pairs, except for the letter's defining contours. As a result, the location of the u-minimum must be due to the interaction of a specific target with its accompanying mask and has no direct relationship with the processing of the information carried by the target.

A clear example of a specific interaction of the target with its mask was found with the " $C$ " target at delay intervals of $20 \mathrm{msec}$ and greater. At those delay intervals, the phenomenal outcome of the target-mask interaction was distinct. The portion of the masking ring adjacent to the break in the " $C$ " was not seen. While the " $C$ " did not have a phenomenally distinct representation, the masking ring appeared as a large " $C$ " with the same orientation as the target. This forward masking of the annular masking figure probably accounted for the improved target identification at the 20- and 30-msec delay intervals. A comprehensive theory of masking will have

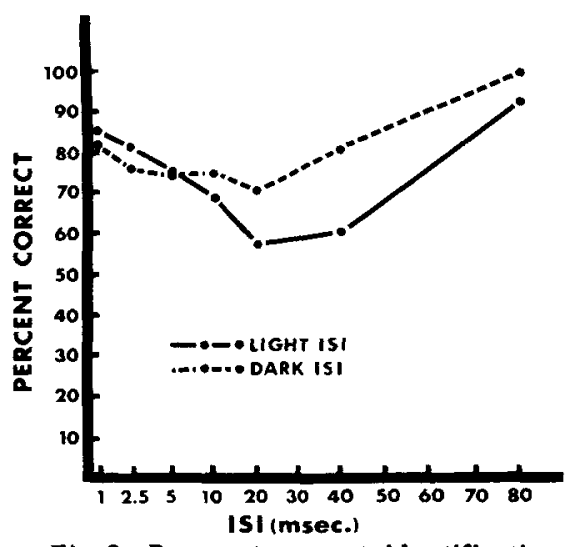

Fig. 3. Per cent correct identification, corrected for chance, of the target pair " $D$ " and " $O$ " when target offset was followed by either a lighted or unlighted ISI. The fixation point was $103.8 \mathrm{~min}$ of arc nasal of the point at which the target was presented.

to account for this forward-masking effect and, in general, predict when backward or forward effects will be observed.

\section{EXPERIMENT 3}

In Experiment 1, masking was obtained only when the masking field contained a masking figure. It is of interest to know whether the duration of the temporal interval separating the target offset and the mask onset is the only important parameter in masking produced with a contoured masking figure or if the homogeneously distributed light energy following target offset does contribute to the masking produced by the masking figure.

The experimental conditions were identical with those of Experiment 2, with the following exceptions: The ISI separating target offset from mask onset was lighted or unlighted; ISI values of 1 , $2.5,5,10,20,40$, and $70 \mathrm{msec}$ were studied; only the " $D$ " and " $O$ " target pair was used.

\section{Results and Discussion}

The masking obtained was a u-shaped function of the ISI $[F(6,24)=8.57$, $\mathrm{p}<.001]$. The amount of masking was greatest for lighted ISIs of $10 \mathrm{msec}$ or more, as Fig. 3 illustrates $[F(1,4)=12.98$, $\mathrm{p}<.025]$. The ability of the lighted ISI to enhance the effect of the masking figure appears to build up as a function of time and does not have any measurable result for delay intervals less than 10 msec under the masking conditions studied in this series of experiments. It can be assumed, though, that the masking function obtained in Experiments 1 and 2 would differ if unlighted ISIs were substituted for the lighted ISIs used.

The luminance values used in generating targets and masks, the duration of the target and mask presentation, and the length of the ISI separating them are the variables most often studied in backward-masking experiments. The present studies extend the number of relevant variables to include target configuration and retinal position stimulated by the target. In addition, it has been shown that even luminance levels of the ISI, which produce no masking effects by themselves, may enhance the masking effects produced by masking figures.

The basic assumption in studying information processing is that errors in the S's performance represent a limitation of his ability to process the information given him. This assumption is not met in backward-masking experiments. The frequency with which errors are made changes with the configuration of the letters used and with the position within the complex array that the letters occupy. Until backward masking is better understood, or until the effects of target contour and position are controlled for, the data from such masking experiments will be of limited value in studying the processing of visual information.

\section{REFERENCES}

AVERBACH, E., \& CORIELL, A. S. Short-term memory in vision. Bell System Technical Journal, 1961, 40, 309-328.

ERIKSEN, C. W. Temporal luminance summation effects in backward and forward masking. Perception \& Psychophysics, 1966, 1, 87-92.

ERIKSEN, C. W., \& COLLINS, J. F. Backward masking in vision. Psychonomic Science, 1964 , 1, 101-102.

ERIKSEN, C. W., COLLINS, J. F., \& GREENSPON, T. S. An analysis of certain factors responsible for nonmonotonic backward masking function. Journal of Experimental Psychology, 1967, 75, 500-507.

KOLERS, P. Intensity and contour effects in visual masking. Vision Research, 1962, 2, 277.294.

LANDAHL, H. D. A neural net model for masking phenomena. Bulletin of Mathematical Biophysics, 1967, 29, 227-232.

LEIBOVIC, $K$. N. A note on visual integrative mechanisms. Bulletin of Mathematical Biophysics, 1965, 27, 305-310.

LEIBOVIC, K. N. Observation on some experiments on visual masking. The Journal of General Psychology, 1968, 78, 19-26.

NEISSER, U. Decision-time without reaction-time: Experiments in visu al scanning. American Journal of Psychology, 1963, 76, 376-385.

NEISSER, U. Cognitive psychology. New York: Appleton-Century-Crofts, 1967.

RAAB, D. H. Backward masking. Psychological Bulletin, 1963, 60, 118-129.

SPERLING, G. A model for visual memory tasks. Human Factors, 1963, 5, 19-31.

STERNBERG, S. Scanning a persisting visual image versus a memorized list. Paper presented 
at the Eastern Psychological Association, 1967.

WEISSTEIN, N. Backward masking and models of perceptual processing. Joumal of Experimental Psychology, 1966, 72, 232-240. WEISSTEIN, N. A Rashevsky-Landah! neural net: Simulation of metacontrast. Psychological
Review, 1968, 75, 494-521.

WEISSTEIN, N., \& HABER, R. N. A u-shaped backward masking function in vision. Psychonomic Science, 1965, 2, 75-76.

WERNER, H. Studies in contour: I. Qualitative analyses. American Journal of Psychology, $1935,47,40-64$.
NOTES

1. Supported by Grant No. NB-07622-02 from the National Institutes of Health.

2. Address: York University, Toronto, Canada.

(Accepted for publication May 21, 1969.) 